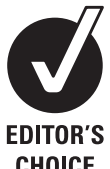

CHOICE

\title{
Rapid sequence induction of anaesthesia in UK emergency departments: a national census
}

Jonathan Benger, ${ }^{1}$ Sarah Hopkinson ${ }^{2}$

${ }^{1}$ Professor of Emergency Care, Faculty of Health and Life Sciences, University of the West of England, Bristol, UK ${ }^{2}$ Academic Clinical Fellow, Academic Department of Emergency Care, University Hospitals Bristol NHS Foundation Trust, Bristol, UK

\section{Correspondence to} Professor J R Benger, Emergency Department, Bristo Royal Infirmary, Marlborough Street, Bristol BS2 8HW, UK; jonathan.benger@uwe.ac.uk

Accepted 7 March 2010 Published Online First 13 December 2010

\begin{abstract}
Introduction Rapid sequence induction of anaesthesia and tracheal intubation (RSI) is an integral part of modern emergency care. Previously, emergency department (ED) RSI has been provided by anaesthetists, but UK emergency physicians are increasingly developing this skill. We undertook a 2-week census of ED RSI to establish a baseline of current practice.
\end{abstract}

Methods All 115 UK College of Emergency Medicine airway leads were contacted and asked to return anonymised data on every drug-assisted intubation occurring in their ED during a 2-week period in September 2008. The number of RSIs and also the total number of $E D$ attendances during the same period were requested.

Results Complete data were returned from 64 EDs $(56 \%)$. The total number of patients undergoing RSI was 218 , with an incidence of $0.12 \%$, or approximately one in every 800 ED attendances. Anaesthetic staff undertook $80 \%$ of ED RSIs, predominantly senior anaesthetic trainees of specialist trainee year 3 (ST3) or above. During normal office hours $74 \%$ of these anaesthetic trainees were supervised during the procedure, with a significant fall in supervision rates to $15 \%$ outside normal office hours ( $p<0.00001$ on $\chi^{2}$ testing).

Discussion The $0.12 \%$ incidence of ED RSI is consistent with previous studies, as is the finding that only $20 \%$ are performed by emergency physicians. The relative infrequency of ED RSI and increasing pool of staff has important implications for training and skills maintenance. Despite the acknowledged difficulty of this technique, nearly half of all ED RSIs are done by unsupervised trainees.

\section{INTRODUCTION}

The UK College of emergency medicine defines emergency medicine as the specialty "which provides immediate care for patients of all ages presenting with illness and injury of all severities'. ${ }^{1}$ The effective delivery of this standard of care requires emergency physicians to possess a wide range of anaesthetic and critical care skills, including those relating to emergency airway management. ${ }^{2}$ Historically, UK emergency physicians have not been trained to deliver the pivotal skill of rapid sequence induction of anaesthesia and tracheal intubation (RSI) in acutely ill and injured patients, relying instead on support from colleagues in anaesthesia and critical care. ${ }^{2}$ However, this can lead to delays in definitive patient management, ${ }^{3}$ and as the specialty of emergency medicine has grown in the UK, increasing numbers of emergency physicians have developed and maintained the skills necessary to undertake RSI independently. ${ }^{4}$
RSI outside the operating theatre is significantly more challenging than routine anaesthesia, with a higher rate of difficult and failed intubation. ${ }^{5}$ It is therefore logical that it should be undertaken by individuals with demonstrated competencies in this area of practice, and junior trainees should be supervised by senior staff. In the UK the exact risks of emergency department (ED) RSI are unknown, as there is no reliable report of the number of patients undergoing RSI in UK EDs, the case mix, indications or staff involved. Data from three EDs in Scotland show an increasing trend towards RSI by emergency physicians, with similar rates of successful intubation and complications to those achieved by anaesthetists. ${ }^{4} 67$ However, a telephone survey of UK EDs reported in 2007 indicated that the majority of ED RSI is still undertaken by anaesthetic staff. ${ }^{8}$

In 2008 the 4th National Audit Project of the Royal College of Anaesthetists (NAP4) was launched. ${ }^{9}$ This set out to determine the incidence of major complications of airway management during anaesthesia. The project collected detailed information on all major airway-related complications in the operating theatre, emergency department and intensive care unit over a 1-year period in the UK. In order to calculate incidence, an accompanying census of all anaesthetic procedures carried out in the operating theatre was completed during 2 weeks in September 2008. We used this opportunity to carry out a similar census of RSIs undertaken in the ED during the same period.

Our aim was therefore to gather reliable information that would inform us of the number of $\mathrm{ED}$ RSIs performed in the UK over a 2-week period, in order to determine the frequency with which RSI is undertaken, the characteristics of patients undergoing RSI and which physicians are performing and supervising this procedure.

\section{METHODS}

The UK College of Emergency Medicine has established a network of 'emergency airway leads' by inviting each UK ED to nominate a consultant representative who acts as a point of contact for matters relating to emergency airway care. Of the 239 major UK EDs, 115 (48\%) currently have a nominated airway lead. These individuals were contacted during September 2008 and invited to collect data on every RSI (defined as emergency tracheal intubation facilitated by drug delivery) occurring in their ED between 00:00 on 15 September and 24:00 on 28 September 2008. Patients who did not need drugs to facilitate intubation were excluded. Each participating ED was asked to return both the total number of patients 
attending the department and the total number undergoing RSI during the 2 -week period, along with an estimate of the accuracy of the latter figure (appendix 1). For those patients undergoing RSI, the date and time of RSI were recorded along with the age and gender of the patient, the indication for RSI and the specialty and grade of the physicians undertaking and supervising the procedure. Doctors were divided into three groups: ST1/2, ST3+ and consultant. Doctors in the ST1/2 grade typically have $1-2$ year's experience in their chosen specialty. ST3+ doctors have three or more year's experience but have not yet achieved consultant status. Emergency medicine ST3+ doctors typically have 6-12 months of anaesthesia and intensive care experience. A doctor was defined as supervising the procedure only if they were identified in this role and physically present in the room at the time of RSI.

Completed data collection forms were returned electronically and by post. Two reminders were sent to encourage return. All data returns were fully anonymised so that individual patients could not be identified. Before analysis, hospital details were removed to ensure anonymity of clinicians and hospitals. Data were entered into an Excel (Microsoft) spreadsheet and analysed using descriptive statistics and $\chi^{2}$ testing with a predetermined significance level of $5 \%$. Subgroup analysis of those patients undergoing RSI following trauma was specified in advance.

\section{RESULTS}

Of the 115 emergency airway leads invited to return data for the census, 64 responded: $56 \%$ of EDs where an emergency airway lead had been identified, and $27 \%$ of all UK EDs. Responses were broadly distributed across England and Scotland, with fewer from Wales and Northern Ireland. However, overall the EDs returning data served the expected proportion of the UK population and comprised a mixture of large and small units. Reported data accuracy was generally high, with $42(66 \%)$ reported as 'accurate' (defined as 0-2\% error) and 14 (22\%) reported as 'close estimate' (2-10\% error).

The total number of patients seen in the participating EDs during the census period was 176036, of whom $218(0.12 \%$ or 1 in 800) underwent RSI. However, since only $10 \%$ of RSIs were done in children, who comprise up to $25 \%$ of total ED attendances, the incidence of ED RSI is considerably lower in children than in adults.

Sixty-six per cent of patients undergoing RSI were male. The distribution of the patients' ages is shown in table 1 . The indications for RSI are shown in table 2.

Seventy-two RSIs (33\%) were carried out during usual office hours (09:00 to 17:00, Monday to Friday). Since this period constitutes $24 \%$ of the total hours in a week, ED RSI is seen to be more common during the hours of the standard working week $\left(\chi^{2}\right.$ test $\left.p=0.03\right)$.

Table 1 Age of patients undergoing rapid sequence induction of anaesthesia in the emergency department

\begin{tabular}{lcc}
\hline Age (years) & Number & Percentage \\
\hline$<1$ & 2 & 1 \\
$1-5$ & 4 & 2 \\
$6-10$ & 5 & 2 \\
$11-17$ & 11 & 5 \\
$18-29$ & 33 & 15 \\
$30-49$ & 59 & 27 \\
$50-69$ & 67 & 31 \\
$70+$ & 33 & 15 \\
Unknown & 4 & 2 \\
Total & 218 & 100 \\
\hline
\end{tabular}

Table 2 Indications for intubation

\begin{tabular}{lcc}
\hline Indication & Number & Percentage \\
\hline Trauma & 54 & 25 \\
Low GCS* & 55 & 25 \\
Respiratory & 39 & 18 \\
Seizure & 20 & 9 \\
Post arrest & 17 & 8 \\
Overdose & 15 & 7 \\
Other & 18 & 8 \\
Total & 218 & 100 \\
\hline *'Low GCS' is due to medical causes, not trauma.
\end{tabular}

GCS, Glasgow Coma Scale.

The specialty and grade of doctor performing the RSI and the rates of direct supervision, divided by whether the RSI took place within or outside of normal office hours, are shown in table 3. No emergency medicine ST1 or ST2 trainee undertook ED RSI during the census period. Direct supervision, when given, was by a consultant in $73 \%$ of cases and a more senior trainee in $27 \%$. Of the consultant supervisors, $23 \%$ were emergency physicians. There is no statistically significant difference in supervision rates between in-office and out-of-office hours for anaesthetic ST1/2 and emergency medicine ST3+ doctors $(p=0.25$ and 0.22 , respectively). Anaesthetic ST3+ doctors were significantly less likely to be supervised outside office hours $(\mathrm{p}<0.00001)$.

Of 54 patients undergoing RSI for trauma, 33 were intubated by an anaesthetist in training (with a supervision rate of $40 \%$ ) and eight by a consultant anaesthetist. Eight were intubated by an emergency physician in training (with a supervision rate of $100 \%$ ) and four by a consultant emergency physician. Data were missing for one patient in this group.

\section{DISCUSSION}

Our census of data relating to RSI performed in UK EDs over a 2 -week period provides a summary of current practice and a baseline against which future developments can be measured. Overall, $83 \%$ of RSIs were performed by trainees and $45 \%$ by unsupervised trainees. Twenty-one per cent of in-hours and $71 \%$ of out-of-hours RSIs were performed by unsupervised trainees.

The incidence of major trauma is declining in the UK. ${ }^{12}$ We found that only $25 \%$ of RSIs are performed in trauma patients, with $75 \%$ of the workload being medical. This is reflected in the age distribution (table 1 ).

The RSI rate of one in 800 patients attending the ED is very similar to that previously reported from a single ED in a district general hospital in Scotland. ${ }^{6}$ On the basis that there are approximately 16 million ED visits annually in the UK, our results equate to $20000 \mathrm{ED}$ RSIs each year. The relative rarity of RSI is an important finding. If an average ED receives 60000 patients per annum, an RSI will be required only once every 5 days. This has implications for training and skill retention if this challenging area of practice is shared between a large pool of trainees and consultants. This is a concern that has been noted by others, ${ }^{810}$ and it will need to be addressed through a range of strategies that develop and maintain effective emergency airway management skills. ${ }^{11}$

We have clearly shown a significant reduction in the supervision of senior anaesthetic trainees undertaking ED RSI outside normal office hours. There was also a proportional decrease in rates of supervision of the more junior anaesthetic and emergency medicine trainees out of hours, though this did not reach statistical significance, perhaps due to their smaller numbers. This is a predictable effect of current working patterns in UK hospitals, in 
Table 3 Rates of direct supervision, according to whether the ED RSI took place inside or outside of normal office hours (9:00 to 17:00 Monday to Friday)

\begin{tabular}{|c|c|c|c|c|}
\hline \multirow[b]{2}{*}{$\begin{array}{l}\text { Grade and specialty of doctor } \\
\text { performing } \mathrm{RSI}^{*}\end{array}$} & \multicolumn{2}{|c|}{ In office hours } & \multicolumn{2}{|c|}{ Out of office hours } \\
\hline & $\begin{array}{l}\text { Number of } \\
\text { RSIs }\end{array}$ & $\begin{array}{l}\text { Number }(\%) \\
\text { supervised }\end{array}$ & $\begin{array}{l}\text { Number of } \\
\text { RSIs }\end{array}$ & $\begin{array}{l}\text { Number }(\%) \\
\text { supervised }\end{array}$ \\
\hline Anaesthetic ST1/2 & 8 & $7(88 \%)$ & 17 & $11(65 \%)$ \\
\hline Anaesthetic ST 3+ & 35 & $26(74 \%)$ & 93 & $14(15 \%)$ \\
\hline Anaesthetic consultant & 13 & - & 8 & - \\
\hline Emergency medicine ST3+ & 9 & $8(89 \%)$ & 18 & $12(67 \%)$ \\
\hline Emergency medicine consultant & 7 & - & 7 & - \\
\hline Total & 72 & $41(79 \%)$ & 143 & $37(29 \%)$ \\
\hline
\end{tabular}

*The senior house officer grade has been merged with specialist trainee (ST) years 1 and 2, while the previous registrar and specialist registrar grade and the staff or trust grade have been merged with ST year $3+$. Data were missing for three patients. - , Supervision not required.

that there are too few consultants to maintain 24-hour presence. However, it does have potential implications for patient safety: recent work from North America has demonstrated a reduced complication rate when senior physicians supervised more junior doctors undertaking emergency intubation. ${ }^{15}$ The apparently reduced rate of ED RSI outside normal working hours may be explained by the fact that fewer patients attend the ED overnight, a different case mix or a reluctance among trainees to undertake RSI in the absence of senior decision-making or direct supervision. This issue requires further research.

ED RSI is a high-risk area, with significantly increased rates of difficult and failed intubation. ${ }^{5}$ RSIs in patients with trauma are often considered particularly difficult, because of the potential for anatomical disruption or blood in the airway, the need to maintain cervical spine immobilisation and the derangement of underlying physiology. ${ }^{13}$ As a result, the need for an emergency surgical airway is more likely. ${ }^{14}$ Despite this, nearly half of all ED RSIs are done by unsupervised trainees, mainly anaesthetic trainees of ST3 grade and above, outside normal office hours. This holds true for RSIs done in trauma patients as well as medical indications.

A limitation of the study is that we were able to obtain detailed data from only $56 \%$ of eligible EDs (27\% all those in the UK). However, the data we did receive from these EDs were of high quality, with a wide geographical spread across England and Scotland. The size of these EDs, in terms of the total number of patients seen in the ED during the census period, also varied considerably, suggesting good representation of both large and small hospitals. However, since we specifically asked senior emergency physicians with a declared interest in emergency airway management to participate, it seems likely that the contribution of emergency medicine to RSI will have been overestimated. Those EDs not returning data are likely to have less active emergency medicine participation in RSI and to rely more on anaesthetists. Therefore it seems reasonable to conclude that no more than $20 \%$ of ED RSIs are currently carried out by emergency physicians, with the bulk of the work $157 \%$ in this survey) being performed by anaesthetic trainees of ST3 grade and above. This is in keeping with the telephone survey reported in 2007, which showed that anaesthetists carried out RSI 'routinely' or 'often' in $81 \%$ of EDs. ${ }^{8}$

Based on the returned census data, it was sometimes difficult to determine the extent to which a trainee was being actively supervised during RSI. For example, a consultant emergency physician leading the care of a trauma patient undergoing RSI by an anaesthetic trainee may have been recorded as the supervising physician but may not have had the skills to undertake RSI personally. The effect of this uncertainty was to overestimate the degree of available supervision. However, in at least 102 of
180 trainee RSIs (57\%) no supervision was recorded, and at least $68 \%$ were not supervised by a consultant. We also assumed that all consultant staff did not require supervision in their practice. Levels of supervision were similar among junior anaesthesia trainees and more senior emergency medicine trainees, perhaps reflecting the fact that emergency airway management is still seen as the province of anaesthetic staff, who are also expected to have more intubation experience than emergency physicians.

In order to improve compliance with the census and the completeness of data return, we did not seek complication rates or patient outcomes. However, this is an important area for future study, along with a more detailed consideration of the drugs and techniques used during ED RSI. NAP4 will examine complications arising from RSI and other airway events in UK EDs and is expected to report during 2010.

\section{CONCLUSIONS}

Approximately one in 800 patients attending a UK ED requires rapid sequence induction of anaesthesia and tracheal intubation, which equates to approximately 20000 patients each year in the UK. Twenty-five per cent of RSIs are required for trauma, and $75 \%$ are for medical indications, most notably a reduced level of consciousness. Fifty-seven per cent of all ED RSIs are done by senior anaesthetic trainees, with a direct supervision rate of $74 \%$ during normal office hours, decreasing to $15 \%$ outside these times. The contribution to ED RSI from emergency physicians remains small, at less than $20 \%$. These findings have important implications for patient safety and for training and skill retention in emergency airway management. They also form a useful benchmark for future developments in practice.

Acknowledgements We would particularly like to acknowledge the 64 College of Emergency Medicine airway leads who collected and returned accurate and comprehensive data for this census, and Jerry Nolan, Dermot McKeown, Tim Cook and Nick Woodall for their comments on the manuscript.The 4th National Audit Project of the Royal College of Anaesthetists (NAP4), led by Tim Cook and Nick Woodall, provided the inspiration and background for this study.

\section{Competing interests None.}

Contributors $\mathrm{JB}$ initiated the study and data collection, analysed the data and drafted the manuscript. SH entered and analysed the data and reviewed the manuscript. JB acts as guarantor.

Provenance and peer review Not commissioned; externally peer reviewed.

\section{REFERENCES}

1. College of Emergency Medicine. The Way Ahead 2008-2012. College of Emergency Medicine: London, 2008. http://www.collemergencymed.ac.uk/CEM/ About $\% 20$ the\%20College/Current $\% 20$ lssues $\% 20$ and $\% 20$ Statements/The $\% 20$ Way\% 20Ahead/default.asp (accessed 16 Apr 2009).

2. Nolan J, Clancy M. Airway management in the emergency department. $\mathrm{Br} \mathrm{J}$ Anaesth 2002;88:9-11. 
3. Boyle AA, Atkinson PRT, Ahmed V, et al. Emergency physician performed rapid sequence induction and system changes reduce time to intubation in critically ill emergency medicine patients. Eur J Emerg Med 2008;15:243-4.

4. Simpson J, Munro PT, Graham CA. Rapid sequence intubation in the emergency department: 5 year trends. Emerg Med J 2006;23:54-6.

5. Mort TC. Emergency tracheal intubation: complications associated with repeated laryngoscopic attempts. Anesth Analg 2004;99:607-13.

6. Stevenson AGM, Graham CA, Hall R, et al. Tracheal intubation in the emergency department: the Scottish district hospital perspective. Emerg Med J 2007:24:394-7

7. Kerslake D, Oglesby AJ, Beard D, et al. Emergency intubation in a scottish urban emergency department: an eight-year analysis. Ann Emerg Med 2008;51:527-8.

8. Dibble CP, McHague C. Rapid sequence intubations by emergency doctors: we can but are we? Emerg Med J 2007;24:480-1.

\section{APPENDIX 1}

\section{Data Collection Form}

Please complete this form for every patient undergoing drug assisted intubation (usually rapid sequence induction of anaesthesia and tracheal intubation: RSI) between 00:01 on Monday September $15^{\text {th }}$ and 23:59 on Sunday September $28^{\text {th }} 2008$.

Completed forms, questions or comments should be returned to: julie.underwood@emergencymedicine.uk.net

\section{Reporting \\ Hospital:}

2. Name and e-mail of College Airway

Link:

3. Total number of patients attending the ED during the two week period:

4. Total number of drug-assisted intubations/RSIs undertaken during the two week period (details below):

5. Please grade the accuracy of the above number (circle one):

Accurate (0-2\% error) Close Estimate (2-10\% error) Estimate $(>10 \%$ error) Guess (an estimate without data to support it)

\section{Detailed Table:}

\begin{tabular}{|c|c|c|c|c|c|c|c|}
\hline No & Date & Time & Age & Sex & $\begin{array}{c}\text { Indication for } \\
\text { intubation/RSI }\end{array}$ & $\begin{array}{c}\text { Grade and } \\
\text { specialty } \\
\text { of } \\
\text { intubating } \\
\text { doctor }\end{array}$ & $\begin{array}{c}\text { Grade and } \\
\text { specialty of } \\
\text { any } \\
\text { supervising } \\
\text { doctor } \\
\text { present }\end{array}$ \\
\hline 1 & & & & & & & \\
\hline 2 & & & & & & & \\
\hline 3 & & & & & & & \\
\hline 4 & & & & & & & \\
\hline 5 & & & & & & & \\
\hline Etc. & & & & & & & \\
\hline
\end{tabular}

9. Benger J. National audit of major airway complications. Emerg Med J 2008.25 (Suppl 1):1. http://emj.bmj.com/cgi/data/25/9/DC1/1 (accessed 16 Apr 2009).

10. Whymark C, Moores A, MacLeod AD. A Scottish National Prospective Study of airway management skills in new-start SHOs. Br J Anaesth 2006;97:473-5.

11. Graham CA. Audit and skills maintenance. In: Benger J, Nolan J, Clancy M, eds. Emergency airway management. Cambridge: Cambridge University Press, 2008:157-60.

12. Lecky F, Woodford M, Yates DW. Trends in trauma care in England and Wales 1989-97. UK Trauma Audit and Research Network. Lancet 2000;355:1771-5.

13. Nee P, Benger J, Walls R. Airway management. Emerg Med J 2008;25:98-102.

14. Reid LA, Ogelsby AJ, Beard D, et al. Surgical airway in the emergency department intubation. Ann Emerg Med 2008;51:528.

15. Schmidt UH, Kumwilaisak K, Bittner E, et al. Effects of supervision by attending anesthesiologists on complications of emergency tracheal intubation. Anesthesiology 2008:109:973-7. 